\title{
Targeted therapy of cancer: new roles for pathologists in colorectal cancer
}

\author{
Stanley R Hamilton \\ Department of Pathology, Division of Pathology and Laboratory Medicine, The University of Texas MD \\ Anderson Cancer Center, Houston, TX, USA
}

\begin{abstract}
Personalized/individualized/tailored therapy for each patient is an important goal for improving the outcome of patients with colorectal adenocarcinoma and includes the intention to maximize efficacy and minimize toxicity of chemotherapeutic agents. Numerous barriers must be overcome to reach this goal because outcome is affected by an unholy trinity of tumor characteristics that include somatic alterations at the DNA, RNA, and protein level; patient characteristics that include germline genetic differences such as polymorphisms in enzymes affecting the metabolism of chemotherapeutic agents; and environmental exposures and factors that include diet and physical activity. At present, evaluation of epidermal growth factor receptor (EGFR) expression by immunohistochemistry in colorectal adenocarcinoma is generally required for treatment with one of the monoclonal antibody therapies directed against that target, despite the absence of evidence for predictive value of the assay, whereas EGFR fluorescent in situ hybridization (FISH) may be predictive. In addition, the Food and Drug Administration of the United States now requires a 'black box' warning on the packaging of irinotecan for evaluation of germline polymorphism in UGT1A1, the gene mutated in Gilbert's syndrome, for potential reduction of drug dosage in patients with the UGT1A1*28 polymorphism. Numerous other potential markers have been identified but have not yet reached levels of evidence that support their routine usage. For example, KRAS gene mutation appears to preclude improved survival after therapy with monoclonal antibody therapy directed at EGFR, and extensive DNA methylation is associated with lack of efficacy of 5-fluorouracil (5-FU)based chemotherapy. Additional markers will come into routine usage as reports of research studies continue to appear in the literature. Clinical trials driven by molecular targets and agents directed against them, and understanding of the conflicting data on utility of markers reported in the literature, are needed to advance the field.
\end{abstract}

Modern Pathology (2008) 21, S23-S30; doi:10.1038/modpathol.2008.14

Keywords: colorectal cancer; targeted therapy; molecular markers; genomics

Targeted therapy consists of therapeutic agents directed at specific molecules. The term is derived from a drug development process that leads to the design and synthesis of a small molecule or production of a monoclonal antibody with specific intent to affect a discreet molecular target in order to have desired therapeutic effects. The term connotes avoidance of the toxicities of cytotoxic chemotherapeutic agents by increased specificity for molecular targets in the context of assumed understanding of the molecular processes and pathways. In practice, most targeted agents have much broader specificity

Correspondence: Dr SR Hamilton, MD, Division of Pathology and Laboratory Medicine-Unit 085, The University of Texas MD Anderson Cancer Center, 1515 Holcombe Boulevard, Box 85, Houston, TX 77030, USA.

E-mail: shamilto@mdanderson.org

Received 11 December 2007; accepted 31 December 2007 than intended, and many cytotoxic agents that are in use after drug development that was based on identification of their functional effects, are actually targeted on specific molecules.

Targeted therapy has the potential to be personalized/individualized/tailored by evaluation of the status of the presumed target and its downstream effector pathways in each patient. ${ }^{1}$ Assessment of tumors for markers useful to personalize cancer therapy is in its infancy, especially in colorectal adenocarcinoma. Markers can be used in two main clinical settings. The first is evaluation of prognosis in which the marker is used to determine the potential need for further treatment based on the natural history and expected behavior of an individual patient's colorectal adenocarcinoma. These markers address the question of whom to treat, especially in the adjuvant setting after an intended curative resection that often leaves the patient cancer-free with no potential for later recurrence of 
his or her resected tumor. The presence of high levels of microsatellite instability (MSI-H) is currently the best example of a favorable prognostic molecular marker in colorectal adenocarcinoma. $^{2,3}$

Identification of patients who have inapparent residual locoregional or distant disease that could be eradicated by therapy would make a major contribution to improving patient management by avoiding the toxicity of unneeded postoperative adjuvant therapy. ${ }^{4-7}$ Molecular staging has been attempted by evaluating tumors for indicators of metastatic phenotype and by evaluating for tumor cell components as molecular evidence of histopathologically inapparent cancer in potential metastatic sites and in blood, other body fluids, and bone marrow as surrogates of micrometastatic disease. Second, predictive markers are of great interest to tailor therapy through use of drugs that are likely to be effective, rather than empirical selection of agents based on published results from series of patients in clinical trials followed by trial-and-error treatment of the individual patient. Predictive markers address the issue of how to treat a patient and have potential applicability in both the adjuvant and advanceddisease settings. ${ }^{8-12}$ These markers would improve patient management by permitting the use of effective agents from the outset of therapy and the avoidance of the toxicities of ineffective agents that are ultimately discontinued due to lack of efficacy after exposure of the patient to potential adverse events. Markers to predict response and, even more importantly, survival would make major changes in patient management.

The development of prognostic and predictive markers in colorectal adenocarcinoma, the second most common cause of cancer deaths in the United States, has a long history, but only a very few markers have reached clinical utility after achieving an acceptable level of evidence. ${ }^{13}$ This review will focus on predictive markers, although many of the markers that are adverse predictive markers are also reported to be adverse prognostic markers, for example, high levels of expression of thymidylate synthase (TS), the target enzyme for 5-fluorouracil (5-FU) that is the oldest and most frequently used agent. The intent of this review is to provide an update on the contributions that pathologists can make in picking the best chemotherapy and targeted therapy for a patient in order to increase the response rate to therapeutic agents in a subset of patients. This approach contrasts with empirical use of agents in all patients, among whom many will have no benefit despite toxicities, and that also dilutes the evidence of effectiveness of the drug in any subgroup with efficacy. Incremental improvements over current approaches to therapy selection are needed and achievable, although major improvements are preferred and more elusive.

Research strategies have included attempts at identification of markers in both tumors themselves and the patients who have tumors. Somatic alterations at the DNA, RNA, and protein levels in tumors are well studied, and comprehensive approaches in characterizing the pharmacogenomics of tumors are being pursued actively. ${ }^{1,8-12,14}$ The germline constitution of patients has also received attention in the discipline of pharmacogenetics with recognition of numerous single-nucleotide polymorphisms in genes that are important in tumor biology. ${ }^{8,15}$ Less effort has been directed at understanding the interaction of environmental and lifestyle exposures and factors such as diet and physical activity with the effects of therapeutic agents, but intriguing results have emerged that western diet and low physical activity have adverse effects on survival after chemotherapy. ${ }^{16,17}$ The unholy trinity of tumor, patient, and environment presents a barrier to progress of translational research, and mechanisms of interactions are poorly understood.

Few clinical trials have been completed to validate the use of predictive markers. The acquisition of patient response rate data with the wide variety of combinations of chemotherapies already available, including both cytotoxic and targeted agents in various dosage schedules, represents a major challenge. Rectal carcinoma is treated with chemoradiation, further complicating the use of markers because some of the mechanisms of effects of radiation differ from those of chemotherapy. ${ }^{18}$ In addition, the molecular pathology of rectal and colonic adenocarcinomas differs. Translation of markers from in vitro to in vivo experimental models and ultimately into patient usage requires many steps in concert with the drug development process. Successful completion of these steps has the additional potential benefit of permitting adjustment of therapy and identification of opportunities for combinations that may not be apparent from empirical drug protocols. Markerguided therapy offers the ability to improve patient selection for treatment with agents, especially if prediction of effects on molecular pathways can be defined. These efforts seem entirely worthwhile, since the five-year survival rate of patients with advanced colorectal adenocarcinoma remains distressing low, even in the current era of available new agents.

\section{Chemotherapeutic agents approved for use in patients with colorectal adenocarcinoma}

Drugs approved for use in colorectal cancer are shown in Table 1 along with their molecular targets and the assays proposed for those targets and markers in single-agent treatment regimens. The agents are the intravenous and oral fluoropyrimidines, 5-FU and capecitabine, respectively; the platinum derivative, oxaliplatin; the camptothecin derivative, irinotecan; the monoclonal antibodies directed 
Table 1 Summary of United States Food and Drug Administration (FDA)-approved agents for treatment of patients with colorectal carcinoma and the associated single-agent tumor and patient markers

\begin{tabular}{|c|c|c|c|c|}
\hline Agent & Type of agent & Target & $\begin{array}{l}\text { Proposed single -agent } \\
\text { tumor markers }\end{array}$ & Proposed patient markers \\
\hline 5-fluorouracil & $\begin{array}{l}\text { Intravenous } \\
\text { fluoropyrimidine }\end{array}$ & $\begin{array}{l}\text { Thymidylate synthase } \\
\text { (TS/TYMS) }\end{array}$ & $\begin{array}{l}\text { Expression of TS, } \\
\text { dihydropyrimidine } \\
\text { dehydrogenase (DPD), } \\
\text { thymidine phosphorylase } \\
\text { (TP) }\end{array}$ & $\begin{array}{l}\text { TS polymorphism, DPD } \\
\text { activity and } \\
\text { polymorphism, } \\
\text { methylene } \\
\text { tetrahydrofolate reductase } \\
\text { polymorphism }\end{array}$ \\
\hline Capecitabine & Oral fluoropyrimidine & TS & ? same as 5 -FU & ? same as 5 -FU \\
\hline Oxaliplatin & Platinum derivative & $\begin{array}{l}\text { Nucleotides in DNA } \\
\text { for crosslinking }\end{array}$ & $\begin{array}{l}\text { Expression of X-ray cross } \\
\text { complementing factor } 1 \\
\text { (XRCC1) and excision } \\
\text { repair cross- } \\
\text { complemenation group } 1 \\
\text { (ERCC1) }\end{array}$ & $\begin{array}{l}\text { XRCC1 and ERCC1 } \\
\text { polymorphism }\end{array}$ \\
\hline Irinotecan & $\begin{array}{l}\text { Camptothecin } \\
\text { derivative }\end{array}$ & $\begin{array}{l}\text { Topoisomerase I } \\
\text { (Topo-1) }\end{array}$ & $\begin{array}{l}\text { ? expression of Topo-1, } \\
\text { ? high levels of } \\
\text { microsatellite instability } \\
(\mathrm{MSI}-\mathrm{H})\end{array}$ & $\begin{array}{l}\text { Uridine diphosphate } \\
\text { glucoronosyltransferase } \\
\text { (UGT1A1) polymorphism }\end{array}$ \\
\hline Bevacizumab & $\begin{array}{l}\text { Humanized monoclonal } \\
\text { antibody }\end{array}$ & $\begin{array}{l}\text { Vascular endothelial } \\
\text { growth factor (VEGF) }\end{array}$ & None identified & None identified \\
\hline Cetuximab & Chimeric IgG1 & $\begin{array}{l}\text { Epidermal growth } \\
\text { factor receptor (EGFR) }\end{array}$ & $\begin{array}{l}\text { Expression of EGFR } \\
\text { (FISH), KRAS mutation }\end{array}$ & None identified \\
\hline Panitumumab & Humanized IgG2 & $\begin{array}{l}\text { Epidermal growth } \\
\text { factor receptor (EGFR) }\end{array}$ & $\begin{array}{l}\text { Expression of EGFR } \\
\text { (FISH), KRAS mutation }\end{array}$ & None identified \\
\hline
\end{tabular}

against epidermal growth factor receptor (EGFR), cetuximab and panitumumab; and the monoclonal antibody directed against vascular endothelial growth factor (VEGF), bevacizumab.

\section{Markers for fluoropyrimidines}

Because intravenous 5 -FU has been in use for decades, the most extensive evaluation of potential markers for sensitivity and resistance to chemotherapy in patients with colorectal adenocarcinoma is available for this drug. TS is the target of 5-FU, and dihydropyrimidine dehydrogenase (DPD) and thymidine phosphorylase (TP) participate in its catabolism. As a result, these enzymes have been studied extensively at the DNA, RNA, and protein levels, and high levels of expression by immunohistochemistry and mRNA associated with poor outcome. ${ }^{1,8-10,19-22}$ Recently, additional enzymes important in 5-FU effects have been identified, including mRNA expression of TNFRSF1B, SLC35F5, and orotate phosphoribosyltransferase. ${ }^{23-25}$

At the DNA level, a tandem repeat of $28 \mathrm{bp}$ is present in the $5^{\prime}$-untranslated region of the TS gene and is linked to its expression and enzymatic activity in tumors. An increase in mRNA and protein has been reported in patients with three repeats (3R) as compared with two repeats (2R). ${ }^{1,26}$ TS copy number has also received attention. ${ }^{27}$ Expression of TS has been evaluated by quantitative reverse transcriptase polymerase chain reaction amplification for identification of mRNA and by immunohistochemistry with a variety of different antibodies. The resulting literature is a quagmire of results in the advanced-disease and adjuvant setting with various chemotherapy regimens, variable methodologies, and, not surprisingly, conflicting results. On the whole, elevated TS expression may be associated with poor response and reduced survival after 5 -FU-based regimens, ${ }^{8,28}$ but many studies have not found the marker to identify responders or survivors, ${ }^{29-31}$ and the current level of evidence does not favor clinical utilization of the assay. ${ }^{13} \mathrm{~A}$ clinical trial in the Eastern Cooperative Oncology Group (protocol E4203) is currently addressing in a prospective manner the potential utility of immunohistochemical expression of TS as an indication for non-fluoropyrimidine-based therapy.

The influence of MSI-H on the response to 5-FU single-agent adjuvant therapy is controversial, with some studies showing no effect on overall survival, ${ }^{2,32}$ but others showing a trend toward $l_{0} e^{2,33}$ or improved survival. ${ }^{2,34}$ A recent study of patients with advanced disease in a phase III clinical trial of 5-FU therapy found that extensive DNA methylation involving CpG islands was associated poor survival, with $97 \%$ of the long-term survivors having a colorectal carcinoma with low or absent methylation. ${ }^{35}$

DPD catabolizes 5-FU, and deficiency in the activity of the gene product predisposes to the development of toxicities. ${ }^{36,37}$ This deficiency is very infrequent, however, and routine testing is not done at present. 
Capecitabine is an oral fluoropyrimidine. ${ }^{38}$ Because it has been in use for far shorter time than 5 -FU, the pharmacogenetics and pharmacogenomics are less well studied. Initial publications suggest that the characteristics of enzymes involved in the metabolism of 5-FU may have similar potential as markers. $^{39}$

\section{Markers for oxaliplatin}

High expression of the excision repair cross-complementing 1 (ERCC1) gene whose product removes oxaliplatin adducts from DNA has been associated with poor outcome after oxaliplatin. ${ }^{28,40,41}$ Increment in the ratio of soluble FAS to FAS ligand/CD95 by enzyme-linked immunosorbent assay in blood after treatment with oxaliplatin and 5-FU combination chemotherapy has been reported as a marker of chemosensitivity in advanced colorectal cancer patients, and decreased ratio as a predictor of chemoresistance. ${ }^{42}$ It seems likely that the finding is a generic effect for platinum agents, rather than specific to oxaliplatin. Favorable germline genotypes from polymorphisms in XPD-751, ERCC1-188, GSTP1-105, and TS-3'-untranslated region have also been associated with improved survival in this setting. ${ }^{43-46}$ Of note, conflicting data on ERCC1-188 have been reported with $\mathrm{C} / \mathrm{C}$ genotype associated with longer survival ${ }^{44}$ and $\mathrm{T} / \mathrm{T}$ genotype associated with higher response rate ${ }^{45}$ in patients with advanced colorectal carcinoma. XRCC1 polymorphism has been associated with worse response. ${ }^{46-48}$

\section{Markers for irinotecan}

Irinotecan is a topoisomerase I inhibitor that is converted to SN-38, the active moiety, by carboxylesterases. This camptothecin derivative has been widely used in combination with 5-FU modulated by leucovorin, with oxaliplatin, and with bevacizumab. ${ }^{49}$ A recent study suggested that patients whose tumor has MSI-H that results from defective mismatch repair gene function have improved survival after treatment with irinotecan. ${ }^{50}$ Germline polymorphism in the uridine diphosphate glucoronosyltransferase (UGT1A1) gene that is mutated in patients with Gilbert's syndrome and participates in irinotecan catabolism is associated with increased toxicity, prompting a Food and Drug Administration (FDA) warning label on the package insert for the drug in the United States. ${ }^{51}$ Genotyping of patients before initiation of irinotecan therapy may become common practice.

\section{Markers for bevacizumab}

This monoclonal antibody against VEGF, in combination with 5-FU/leucovorin or irinotecan and 5-FU/leucovorin, improves survival of patients with advanced colorectal cancer. ${ }^{52}$ Despite extensive efforts, predictive markers have not been identified $^{53-56}$ in tumors, blood, or circulating tumor cells and endothelial cells.

\section{Markers for antibodies to EGFR}

Although demonstration of EGFR in a tumor would seem logically to be required for effective targeted therapy with agents targeting the gene product, several studies have shown no relationship of immunohistochemical expression in single-agent therapy with cetuximab and combination therapy of cetuximab with irinotecan in patients with advanced disease, ${ }^{57}$ or with single-agent panitumumab. ${ }^{58,59}$ By contrast, overexpression of EGFR and of HER2 by fluorescent in situ hybridization (FISH) may be predictive of response, ${ }^{60-62}$ although these results are controversial, ${ }^{63}$ perhaps due to lack of standardization of methods. ${ }^{64}$ In single-agent therapy with cetuximab, low expression of EGFR, cyclooxygenase 2, and interleukin-8 mRNA was associated with improved overall survival, and high expression of VEGF mRNA with resistance in patients with advanced refractory disease. ${ }^{65}$ Germline polymorphism of the cyclin D gene and gene expression levels of VEGF have been reported to be associated with efficacy of cetuximab. ${ }^{28}$ Recent data have shown that patients with KRAS protooncogene mutation in their tumor have no improvement in survival after treatment with cetuximab or panitumumab. ${ }^{66,67}$ These findings have biologic plausibility because KRAS is downstream of EGFR in signal transduction pathways, such that activating mutation of KRAS would replace the dependency of the tumor cells on increased signaling from upstream EGFR.

\section{Markers in combination therapy regimens}

Use of combination chemotherapy is standard practice, but poses substantial challenges for the use of markers because of the various mechanisms of action of cytotoxic and targeted agents. Studies have begun to address combination therapies. Germline polymorphisms of TS, XRCC1, and UGT1A1 were evaluated in patients with advanced colorectal cancer treated with 5-FU and irinotecan or 5-FU and oxaliplatin. ${ }^{68}$ With the latter regimen, patients with TS $5^{\prime}$ single-nucleotide polymorphism and/or favorable XRCC1 genotypes had better time to progression. With the combination of capecitabine and irinotecan, patients whose tumor had TP expression by immunohistochemistry had improved overall survival, whereas TS and DPD were not predictive. ${ }^{69}$ High expression of ERCC1 and TS mRNA in patients with advanced colorectal cancer treated with 5-FU and oxaliplatin has been associated with poorer survival. ${ }^{70}$ In rectal cancer patients treated with chemoradiation with a 5-FU 
regimen, high intratumoral TS after therapy was reported to be predictive of unfavorable outcome. ${ }^{71}$

\section{Challenges for development of clinically usable markers}

Despite the progress that has been made in the development of markers for use in therapeutics, many clinical, biological, and logistical hurdles remain for the markers to be used in patient management. In the clinical arena, the low response rates for many agents make evaluation of markers difficult due to the small number of patients with favorable outcome who enter into statistical analysis. In addition, agents are routinely used in combination, often with variable dosage schedules (eg, IFL or FOLFIRI for combinations of 5-FU with irinotecan). Agents from different pharmaceutical and biotechnology companies are often combined in regimens, leading to concerns about intellectual property and data sharing. Validation in prospective clinical trials remains the gold standard for levels of evidence to support use of a marker in clinical practice. Resources are limited, however, for carrying out such trials, especially with the large number of opportunities provided by the development of numerous promising new agents and the permutations and combinations of new and established agents. Finally, the rational selection of markers and development of robust methodologies for their use is difficult, as some markers can be evaluated at the DNA, RNA, and protein levels using various technologies. ${ }^{64}$ The decision on the best method and definition of its performance characteristics in the clinical arena require sophisticated clinical, laboratory, and statistical approaches.

Biological challenges to the development of markers include the complexity of the cellular mechanisms of intrinsic and acquired sensitivity and resistance in tumors. Differing mechanisms of effects are typical of different therapeutic agents, including both targeted and cytotoxic agents. Intratumor heterogeneity comes into play, as tumors consist of neoplastic cells in a complex microenvironment of non-neoplastic host cells, including new blood vessels resulting from angiogenesis, stromal cells, and inflammatory cells. The targets of some agents are not the tumor cells, but rather the host cells (eg, antivascular/anti-angiogenesis therapy), requiring assessment of localization of markers that cannot be accomplished by 'grind and bind' methodologies that fail to preserve topography. Finally, trafficking of signals through complex interacting molecular pathways is typical, and targeted agents often have effects in multiple pathways because of homologies, including downstream pathways as well as the targets themselves, due to subtotal specificity. In fact, some studies suggest that 'dirty' agents with broader effects are superior to agents with high target specificity.
Studies to address markers also have logistical challenges. The availability of appropriate tissue and body fluid repositories as well as collection and distribution of the specimens to research laboratories are complicated. These logistics must be accomplished in concert with data management, bioinformatics, and biostatistics. Retrospective studies are easier to conduct and provide faster answers due to the availability of existing specimens and outcome data. Temporal trends, however, in the biology of various tumor types and their clinical characteristics (eg, improved survival of stage II and III colon cancer patients in recent years) can affect the validity of the studies. New therapeutic regimens are emerging constantly, and prospective studies are difficult to design and complicated to carry out. The attitude in the gastrointestinal medical oncology community to apply markers for stratification and treatment assignment of patients in clinical trials lags far behind that of medical oncologists who deal with breast cancer patients. ${ }^{72}$ In addition, the cost of evaluating markers increases the expense of drug development, although most pharmaceutical companies now use the strategy of developing markers in parallel with their agents.

New technologies will have major impact on marker development. In the '-omics' era, broad-scale analysis of genes in genomics and methylomics, and of their RNA and products in transcriptomics, proteomics, and metabolomics is underway. Noncoding regulatory RNAs, including microRNAs, offer another opportunity for marker development. Broad-scale studies will provide substantial information that can be developed into clinically useful markers.

Continuing progress will depend upon meeting challenges to marker development. Aligning combination chemotherapy regimens and panels of markers must occur. Better understanding of the tumor biology and patient biology that underlies intrinsic resistance to therapy and the acquisition of resistance after treatment will provide a rationale basis for markers that should be developed. The heterogeneity of marker applications, such as differences between the advanced-disease and adjuvant clinical settings, requires critical consideration of the intent of marker development. Finally, crucial but mundane research directed at standardizing methodologies for marker testing must be conducted, especially since many targets can be evaluated by multiple methods for multiple analyte forms, for example, DNA, RNA, and protein by sequence analysis, epigenetics, transcriptome microarrays, in situ hybridization, immunohistochemistry, posttranslational modification, and so on.

\section{Current status}

At present, evaluation of EGFR expression by immunohistochemistry in colorectal adenocarcinoma 
is generally required for treatment with one of the monoclonal antibodies directed against that target, despite the absence of evidence for predictive value of the assay. Evaluation of EGFR overexpression by FISH may be predictive. In addition, other markers are directed at toxicity of agents. The FDA of the United States now requires a 'black box' warning on the packaging of irinotecan for evaluation of germline UGT1A1 polymorphism for potential reduction of drug dosage in patients who have the UGT1A 1 * 28 polymorphism. Low-level or absence of DPD that catabolizes 5-FU is associated with 5-FU toxicity, but the abnormality is so uncommon that testing is very rarely performed before therapy. Numerous other potential markers have been identified but have not yet reached levels of evidence that support their routine usage. Clinical trials remain as the preferred source of data to provide the needed evidence, and multivariable approaches through the use of panels of markers are required in the current era of combination therapies.

\section{Acknowledgements}

This manuscript was prepared by Cheryl Willis.

\section{Disclosure/conflict of interest}

Dr Hamilton is the recipient of research funds from Genentech and a paid consultant for Novartis.

\section{References}

1 Danenberg PV. Pharmacogenomics of thymidylate synthase in cancer treatment. Front Biosci 2004;9: 2484-2494.

2 Popat S, Hubner R, Houlston RS. Systematic review of microsatellite instability and colorectal cancer prognosis. J Clin Oncol 2005;23:609-618.

3 Funaioli C, Pinto C, Mutri V, et al. Does biomolecular characterization of stage II/III colorectal cancer have any prognostic value? Clin Colorectal Cancer 2006;6:38-45.

4 Chau I, Cunningham D. Adjuvant therapy in colon cancer-what, when and how? Ann Oncol 2006;17: 1347-1359.

5 Benson III AB. Adjuvant chemotherapy of stage III colon cancer. Semin Oncol 2005;32:S74-S77.

6 Pallis AG, Mouzas IA. Adjuvant chemotherapy for colon cancer. Anticancer Res 2006;26:4809-4815.

7 Benson III AB. New approaches to the adjuvant therapy of colon cancer. Oncologist 2006;11:973-980.

8 Ahmed FE. Molecular markers that predict response to colon cancer therapy. Expert Rev Mol Diagn 2005;5:353-375.

9 Allen WL, Johnston PG. Have we made progress in pharmacogenomics? The implementation of molecular markers in colon cancer. Pharmacogenomics 2005;6:603.

10 Lenz HJ. Pharmacogenomics and colorectal cancer. Lopez-Guerrero JA (ed). New Trends in Cancer for the 21st Century. Springer: New York, 2006, pp 211-231.
11 Holen KD. Target practice: figuring out which, when, and why to use systemic therapies for metastatic colon cancer. Cancer Invest 2006;24:98-105.

12 Allen WL, Coyle VM, Johnston PG. Predicting the outcome of chemotherapy for colorectal cancer. Curr Opin Pharmacol 2006;6:332-336.

13 Locker GY, Hamilton S, Harris J, et al. ASCO 2006 update of recommendations for the use of tumor markers in gastrointestinal cancer. J Clin Oncol 2006;24:5313-5327.

14 Russo A, Corsale S, Cammareri P, et al. Pharmacogenomics in colorectal carcinomas: future perspectives in personalized therapy. J Cell Physiol 2005;204:742-749.

15 Saito YA, Camilleri M. Clinical application of pharmacogenetics in gastrointestinal diseases. Expert Opin Pharmacother 2006;7:1857-1869.

16 Meyerhardt JA, Niedzwiecki D, Hollis D, et al. Association of dietary patterns with cancer recurrence and survival in patients with stage III colon cancer. J Am Med Assoc 2007;298:754-764.

17 Meyerhardt JA, Heseltine D, Niedzwiecki D, et al. Impact of physical activity on cancer recurrence and survival in patients with stage III colon cancer: findings from CALGB 89803. J Clin Oncol 2006;24: 3517-3518.

18 Ochler C, Ciernik IF. Radiation therapy and combined modality treatment of gastrointestinal carcinomas. Cancer Treat Rev 2006;32:119-138.

19 Ciaparrone M, Quirino M, Schinzari G, et al. Predictive role of thymidylate synthase, dihydropyrimidine dehydrogenase and thymidine phosphorylase expression in colorectal cancer patients receiving adjuvant 5-fluorouracil. Oncology 2006;70:366-377.

20 Jensen SA, Vainer B, Sorensen JB. The prognostic significance of thymidylate synthase and dihydropyrimidine dehydrogenase in colorectal cancer of 303 patients adjuvantly treated with 5-fluorouracil. Int J Cancer 2007;120:694-701.

21 Salonga D, Danenberg KD, Johnson M, et al. Colorectal tumors responding to 5-fluorouracil have low gene expression levels of dihydropyrimidine dehydrogenase, thymidylate synthase, and thymidine phosphorylase. Clin Cancer Res 2000;6:1322-1327.

22 Grem JL. Intratumoral molecular or genetic markers as predictors of clinical outcome with chemotherapy in colorectal cancer. Semin Oncol 2005;32: 120-127.

23 Matsuyama R, Togo S, Shimizu D, et al. Predicting 5fluorouracil chemosensitivity of liver metastases from colorectal cancer using primary tumor specimens: Three-gene expression model predicts clinical response. Int J Cancer 2006;119:406-413.

24 Matsusaka S, Yamasaki H, Fukushima $\mathrm{M}$, et al. Upregulation of enzymes metabolizing 5-fluorouracil in colorectal cancer. Chemotherapy 2007;53:36-41.

25 Iacopetta B. Methyl-group metabolism and the response of colorectal cancer to 5-fluorouracil. Crit Rev Oncog 2006;12:115-126.

26 Marsh S. Thymidylate synthase pharmacogenetics. Invest New Drugs 2005;23:533-537.

27 Brody JR, Huel T, Gallmeier E, et al. Genomic copy number changes affecting the thymidylate synthase (TYMS) gene in cancer: a model for patient classification to aid fluoropyrimidine therapy. Cancer Res 2006;66:9369-9373.

28 Lenz HJ. Pharmacogenomics and colorectal cancer. Adv Exp Med Biol 2006;587:211-231. 
29 Sinicrope FA, Rego RL, Halling KC, et al. Thymidylate synthase expression in colon carcinomas with microsatellite instability. Clin Cancer Res 2006;12: 2738-2744.

30 Noda E, Maeda K, Inoue T, et al. Expression of genes for 5-FU-metabolizing enzymes and response to irinotecan plus 5-FU-leucovorin in colorectal cancer. Anticancer Res 2006;26:4653-4658.

31 Popat S, Chen Z, Zhao D, et al. A prospective, blinded analysis of thymidylate synthase and p53 expression as prognostic markers in the adjuvant treatment of colorectal cancer. Ann Oncol 2006;17:1810-1817.

32 Kim GP, Colangelo LH, Wieand HS, et al. Prognostic and predictive roles of high-degree microsatellite instability in colon cancer: a National Cancer Institute-National Surgical Adjuvant Breast and Bowel Project collaborative study. J Clin Oncol 2007;25: 767-772.

33 Ribic CM, Sargent DJ, Moore MJ, et al. Tumor microsatellite-instability status as a predictor of benefit from fluorouracil-based adjuvant chemotherapy for colon cancer. N Engl J Med 2003;349:247-257.

34 Elsaleh H, Powell B, McCaul K, et al. P53 alteration and microsatellite instability have predictive value for survival benefit from chemotherapy in stage III colorectal carcinoma. Clin Cancer Res 2001;7:1343-1349.

35 Shen L, Catalano PJ, Benson III AB, et al. Association between DNA methylation and shortened survival in patients with advanced colorectal cancer treated with 5-fluorouracil-based chemotherapy. Clin Cancer Res 2007;13:6093-6098.

36 van Kuilenburg AB. Screening for dihydropyrimidine dehydrogenase deficiency: to do or not to do, that's the question. Cancer Invest 2006;24:215-217.

37 Ploylearmsaeng SA, Fuhr U, Jetter A. How may anticancer chemotherapy with fluorouracil be individualized? Clin Pharmacokinet 2006;45:567-592.

38 Schmoll HJ, Arnold D. Update on capecitabine in colorectal cancer. Oncologist 2006;11:1003-1009.

39 Salgado J, Zabalegui N, Gil C, et al. Polymorphisms in the thymidylate synthase and dihydropyrimidine dehydrogenase genes predict response and toxicity to capecitabine-raltitrexed in colorectal cancer. Oncol Rep 2007;17:324-328.

40 Lentz F, Tran A, Rey E, et al. Pharmacogenomics of fluorouracil, irinotecan, and oxaliplatin in hepatic metastases of colorectal cancer. Am J Pharmacogenomics 2005;5:21-33.

41 Gossage L, Madhusudan S. Current status of excision repair cross complementing-group 1 (ERCC1) in cancer. Cancer Treat Rev 2007;33:565-577.

42 Nadal C, Maurel J, Gallego R, et al. FAS/FAS ligand ratio: a marker of oxaliplatin-based intrinsic and acquired resistance in advanced colorectal cancer. Clin Cancer Res 2005;11:4770-4774.

43 Stoehlmacher J, Park DJ, Zhang W, et al. A multivariate analysis of genomic polymorphisms: prediction of clinical outcome to 5-FU/oxaliplatin combination chemotherapy in refractory colorectal cancer. $\mathrm{Br}$ J Cancer 2004;91:344-354.

44 Park DJ, Zhang W, Stochlmacher J, et al. ERCC1 gene polymorphism as a predictor for clinical outcome in advanced colorectal cancer patients treated with platinum-based chemotherapy. Clin Adv Hematol Oncol 2003;1:162-166.

45 Viguier J, Boige V, Miguel C, et al. ERCC1 codon 118 polymorphism is a predictive factor for the tumor response to oxaliplatin/5-fluorouracil combination chemotherapy in patients with advanced colorectal cancer. Clin Cancer Res 2005;11:6212-6217.

46 Suh KW, Kim JH, Kim DY, et al. Which gene is a dominant predictor of response during FOLFOX chemotherapy for the treatment of metastatic colorectal cancer, the MTHFR or XRCC1 gene? Ann Surg Oncol 2006;13:1379-1385.

47 Ruzzo A, Graziano F, Loupakis F, et al. Pharmacogenetic profiling in patients with advanced colorectal cancer treated with first-line FOLFOX-4 chemotherapy. J Clin Oncol 2007;25:1247-1254.

48 Martinez-Balibrea E, Manzano JL, Martinez-Cardus A, et al. Combined analysis of genetic polymorphisms in thymidylate synthase, uridine diphosphate glucoronosyltransferase and X-ray cross complementing factor 1 genes as a prognostic factor in advanced colorectal cancer patients treated with 5-fluorouracil plus oxaliplatin or irinotecan. Oncol Rep 2007;17:637-645.

49 Fuchs C, Mitchell EP, Hoff PM. Irinotecan in the treatment of colorectal cancer. Cancer Treat Rev 2006;32:491-503.

50 Bertagnolli MM, Compton CC, Niedzwiecki D, et al. Microsatellite instability predicts improved response to adjuvant therapy with irinotecan, 5-fluorouracil and leucovorin in stage III colon cancer. J Clin Oncol 2006;24: abstract 10003 .

51 Nguyen H, Tran A, Lipkin S, et al. Pharmacogenomics of colorectal cancer prevention and treatment. Cancer Invest 2006;24:630-639.

52 Marshall J. The role of bevacizumab as first-line therapy for colon cancer. Semin Oncol 2005; 32(6 Suppl 9):S43-S47.

53 Diaz-Rubio E. Vascular endothelial growth factor inhibitors in colon cancer. Adv Exp Med Biol 2006;587:251-275.

54 Jubb AM, Hurwitz HI, Bai W, et al. Impact of vascular endothelial growth factor-A expression, thrombospondin-2 expression, and microvessel density on the treatment effect of bevacizumab in metastatic colorectal cancer. J Clin Oncol 2006;24: 217-227.

55 Ince WL, Jubb AM, Holden SN, et al. Association of k-ras, b-raf, and p53 status with the treatment effect of bevacizumab. J Natl Cancer Inst 2005;97:981-989.

56 Jubb AM, Oates AJ, Holden S, et al. Predicting benefit from anti-angiogenic agents in malignancy. Nature 2006;6:626-635.

57 Hebbar M, Wacrenier A, Desauw C, et al. Lack of usefulness of epidermal growth factor receptor expression determination for cetuximab therapy in patients with colorectal cancer. Anticancer Drugs 2006;17: 855-857.

58 Chua YJ, Cunningham D. Panitumumab. Drugs Today 2006;42:711-719.

59 Wainberg Z, Hecht JR. Panitumumab in colon cancer: a review and summary of ongoing trials. Expert Opin Biol Ther 2006;6:1229-1235.

60 Cappuzzo F, Finocchiaro G, Rossi E, et al. EGFR FISH assay predicts for response to cetuximab in chemotherapy refractory colorectal cancer patients. Ann Oncol 2007, Oct 31; e-pub ahead of print.

61 Moroni M, Veronese S, Benvenuti S, et al. Gene copy number for epidermal growth factor receptor (EGFR) and clinical response to antiEGFR treatment in colorectal cancer: a cohort study. Lancet Oncol 2005;6: 257-286. 
62 Sartore-Bianchi A, Moroni M, Veronese S, et al. Epidermal growth factor receptor gene copy number and clinical outcome of metastatic colorectal cancer treated with panitumumab. J Clin Oncol 2007;25:3238-3245.

63 Italiano A, Follana P, Caroli F-X, et al. Cetuximab shows activity in colorectal cancer patients with tumors for which FISH analysis does not detect an increase in EGFR gene copy number. Ann Surg Oncol 2007, Nov 7; e-pub ahead of print.

64 Spindler KL, Lindebjerg J, Nielsen JN, et al. Epidermal growth factor receptor analyses in colorectal cancer: a comparison of methods. Int J Oncol 2006;29:1159-1165.

65 Vallbohmer D, Zhang W, Gordon M, et al. Molecular determinants of cetuximab efficacy. J Clin Oncol 2005;23:3536-3544.

66 Finocchiaro G, Cappuzzo F, Janne PA, et al. EGFR, HER2 and Kras as predictive factors for cetuximab sensitivity in colorectal cancer. J Clin Oncol 2007;25:168s, abstract 4021.

67 De Roock W, Piessevaux H, De Schutter J, et al. KRAS wild-type state predicts survival and is associated to early radiological response in metastatic colorectal cancer treated with cetuximab. Ann Oncol 2007, Nov 12; e-pub ahead of print.

68 Martinez-Balibrea E, Manzano JL, Martinez-Cardus A, et al. Combined analysis of genetic polymorphisms in thymidylate synthase, uridine diphosphate glucoronosyltransferase and X-ray cross complementing factor 1 genes as a prognostic factor in advanced colorectal cancer patients treated with 5-fluorouracil plus oxaliplatin or irinotecan. Oncol Rep 2007;17: 637-645.

69 Meropol NJ, Gold PJ, Diasio RB, et al. Thymidine phosphorylase expression is associated with response to capecitabine plus irinotecan in patients with metastatic colorectal cancer. J Clin Oncol 2006;24: 4069-4077.

70 Shirota Y, Stoehlmacher J, Brabender J, et al. ERCC1 and thymidylate synthase mRNA levels predict survival for colorectal cancer patients receiving combination oxaliplatin and fluorouracil chemotherapy. J Clin Oncol 2001;19:4298-4304.

71 Liersch T, Langer C, Ghadimi BM, et al. Lymph node status and TS gene expression are prognostic markers in stage II/III rectal cancer after neoadjuvant fluorouracil-based chemoradiotherapy. J Clin Oncol 2006;24: 4062-4068.

72 Love N, Bylund C, Meropol NJ, et al. How well do we communicate with patients concerning adjuvant systemic therapy? A survey of 150 colorectal cancer survivors. J Clin Oncol 2007;25:168s, abstract 4020 . 